\title{
The potential role of glutamate transporters in the pathogenesis of normal tension glaucoma
}

\author{
Takayuki Harada, ${ }^{1,2,3}$ Chikako Harada,, ${ }^{1,2}$ Kazuaki Nakamura, ${ }^{2}$ Hun-Meng A. Quah,1 \\ Akinori Okumura, ${ }^{2}$ Kazuhiko Namekata, ${ }^{2}$ Tadashiro Saeki, ${ }^{4}$ Makoto Aihara, ${ }^{4}$ \\ Hiroshi Yoshida, ${ }^{3}$ Akira Mitani, ${ }^{5}$ and Kohichi Tanaka ${ }^{1,6,7}$
}

\begin{abstract}
${ }^{1}$ Laboratory of Molecular Neuroscience, School of Biomedical Science and Medical Research Institute, Tokyo Medical and Dental University, Tokyo, Japan. ${ }^{2}$ Department of Molecular Neurobiology, Tokyo Metropolitan Institute for Neuroscience, Fuchu, Japan.

${ }^{3}$ Department of Neuro-ophthalmology, Tokyo Metropolitan Neurological Hospital, Fuchu, Japan. ${ }^{4}$ Department of Ophthalmology, University of Tokyo School of Medicine, Tokyo, Japan. ${ }^{5}$ Human Health Science, Graduate School of Medicine, Kyoto University, Kyoto, Japan. ${ }_{6}^{6}$ Precursory Research for Embryonic Science and Technology, Japan Science and Technology Agency, Kawaguchi, Japan. ${ }^{7}$ Center of Excellence Program for Brain Integration and its Disorders, Tokyo Medical and Dental University, Tokyo, Japan.
\end{abstract}

\begin{abstract}
Glaucoma, a progressive optic neuropathy due to retinal ganglion cell (RGC) degeneration, is one of the leading causes of irreversible blindness. Although glaucoma is often associated with elevated intraocular pressure (IOP), IOP elevation is not detected in a significant subset of glaucomas, such as normal tension glaucoma (NTG). Moreover, in some glaucoma patients, significant IOP reduction does not prevent progression of the disease. Thus, understanding IOP-independent mechanisms of RGC loss is important. Here, we show that mice deficient in the glutamate transporters GLAST or EAAC1 demonstrate spontaneous RGC and optic nerve degeneration without elevated IOP. In GLAST-deficient mice, the glutathione level in Müller glia was decreased; administration of glutamate receptor blocker prevented RGC loss. In EAAC1-deficient mice, RGCs were more vulnerable to oxidative stress. These findings suggest that glutamate transporters are necessary both to prevent excitotoxic retinal damage and to synthesize glutathione, a major cellular antioxidant and tripeptide of glutamate, cysteine, and glycine. We believe these mice are the first animal models of NTG that offer a powerful system for investigating mechanisms of neurodegeneration in NTG and developing therapies directed at IOP-independent mechanisms of RGC loss.
\end{abstract}

\section{Introduction}

It is estimated that glaucoma affects nearly 70 million individuals worldwide, with at least 6.8 million bilaterally blind (1). The disease is characterized by a slow progressive degeneration of retinal output neurons (retinal ganglion cells [RGCs]) and their axons, which is usually associated with elevated intraocular pressure (IOP). The common adult-onset glaucoma is primary open-angle glaucoma (POAG), which is probably caused by a reduction in outflow of aqueous humor through the trabecular outflow pathways (2). Normal tension glaucoma (NTG), a subset of POAG that indicates statistically normal IOP, also shows glaucomatous optic neuropathy and relevant visual field defect. Several population studies have suggested that NTG represents $20 \%-90 \%$ of all POAG, with percentages seeming to vary according to race (3-5). Interestingly, IOP still seems to play a role in NTG because a substantial number of patients with NTG as well as other forms of POAG benefit from lowering of IOP (6). Thus, NTG may be caused by the vulnerability of optic nerves to normal range of IOP. However, it should be noted that some of NTG patients are still progressive in spite of sufficient IOP reduction. All these observations suggest a possibility that factors not dependent on IOP may contribute to disease progress

Nonstandard abbreviations used: EAAC1, excitatory amino acid carrier 1; GCL, ganglion cell layer; GLAST, glutamate/aspartate transporter; GLT-1, glutamate transporter 1; GS, glutamine synthetase; INL, inner nuclear layer; IOP, intraocular pressure; $2 \mathrm{~K}$, second-order kernel; mfERG, multifocal electroretinogram; NTG, normal tension glaucoma; ONL, outer nuclear layer; POAG, primary open-angle glaucoma; RGC, retinal ganglion cell.

Conflict of interest: The authors have declared that no conflict of interest exists. Citation for this article: J. Clin. Invest. 117:1763-1770 (2007). doi:10.1172/JCI30178. and that elucidating such factors would be necessary to understand the pathogenesis of glaucoma and guide efforts toward improved therapeutics.

Recent studies have shown that glaucoma is affected by multiple genetic and environmental factors $(7,8)$, and this complexity can make it difficult to reach definitive conclusions (especially in human studies, in which many factors cannot be controlled). Animal studies complement human studies and can provide important insights into genetic etiology and molecular mechanisms for further assessment in people. There are now several published mouse models of pressure-induced RGC death. These include inherited and experimentally induced models (9). However, no animal model for NTG is currently known and available for research.

Besides more extensively investigated factors, such as reduced ocular blood flow, ocular vascular dysregulation, and systemic blood pressure alterations, excessive stimulation of the glutamatergic system, specifically the NMDA subtypes, has been proposed to contribute to death of RGCs in glaucoma. Glutamate transporter is the only mechanism for the removal of glutamate from the extracellular fluid in the retina (10). Therefore, it is hypothesized that the increase in glutamate may result from a failure of the glutamate transporters adjacent to RGCs. In the inner plexiform layer where synapses on RGCs exist, there are 3 transporters involved in this task: glutamate transporter 1 (GLT-1), located in the bipolar cell terminals; excitatory amino acid carrier 1 (EAAC1) in retinal neurons including RGCs; and glutamate/aspartate transporter (GLAST) in Müller cells (11). However, there is still debate on whether excitotoxic damage is involved in the pathophysiology of glaucoma (12). Here, we utilized mice in which 1 of each of these 3 glutamate transporters has been knocked out 

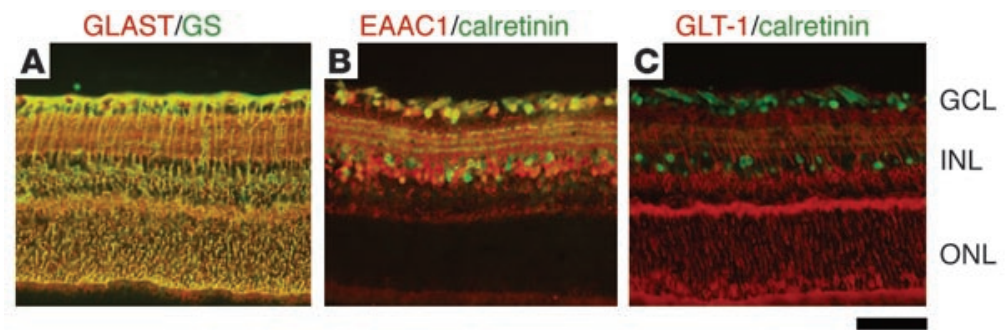

\section{Figure 1}

Expression of glutamate transporters in the retina. Immunohistochemical analysis of mouse retina doublestained with GLAST and GS, a specific marker for Müller glial cells (A); EAAC1 and calretinin, a specific marker for RGCs and amacrine cells (B); and GLT-1 and calretinin antibodies (C). Scale bar: $50 \mu \mathrm{m}$. and examined the long-term effect of retinal morphology during postnatal development on RGC survival in vivo. We found that GLAST- and EAAC1-knockout mice showed spontaneously occurring RGC death and typical glaucomatous damage of the optic nerve without elevated IOP. To our knowledge, these glutamate transporter knockout mice are the first animal models of NTG, which offer a powerful system for determining mechanisms and evaluating new treatments of NTG.

\section{Results}

Degeneration of RGCs in GLAST- and EAAC1-deficient mice. Immunohistochemical studies demonstrated the presence of 3 subtypes of glutamate transporters in the inner plexiform layer of the rat retina. GLAST is expressed on Müller cells, GLT-1 on the bipolar cell terminals, and EAAC1 on ganglion cells (11). We verified that these localizations are also true for the mouse. As we previously reported (13), GLAST immunoreactivity is present throughout the retina and double-labeled by glutamine synthetase (GS), a specific marker for Müller glial cells (Figure 1A). EAAC1 and GLT-1 are neural-type glutamate transporters; however, their distribution is different. EAAC1 is mainly localized to the inner retinal layer and double-labeled by calretinin, a specific marker for RGCs and amacrine cells (Figure 1B). On the other hand, GLT-1 immunoreactivity is present in a small fraction of photoreceptors in the outer nuclear layer (ONL) in addition to bipolar cells but not in RGCs (Figure 1C). The findings are in agreement with those reported by Rauen for rat retina (11). The retinae of GLAST (14) and EAAC1 (15) mutant mice have normal organization at birth (Figure 2A). However, we noticed severe retinal degeneration in 8-month-old GLAST ${ }^{-/-}$mice (see also Figure 3, A and B), in

\section{Figure 2}

RGC degeneration in glutamate transporter mutant mice. (A) H\&E staining of retinal sections during postnatal development. WT, GLAST+/-, and GLAST $^{-/-}$mice are littermates. $\mathrm{EAAC}^{+/-}$and $\mathrm{EAAC}^{-/-}$mice are littermates. (B) Quantification of RGC number in glutamate transporter mutant mice. The number of neurons in the GCL was counted in the retinal section from one ora serrata through the optic nerve to the other ora serrata at 0,1 , $2,3,5,8,16$, and 32 weeks of age. Each plot represents the results of 3 to 6 independent experiments. Scale bar: $50 \mu \mathrm{m}$ (A). NBL, neuroblast layer.
A
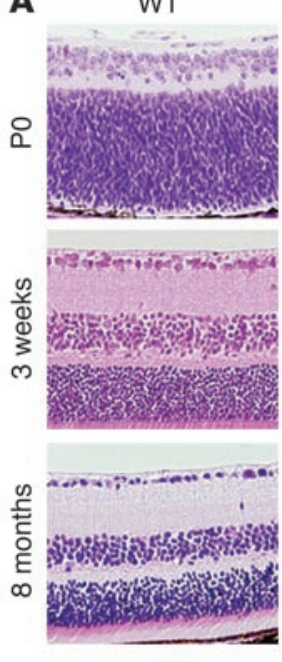

GLAST $^{-1-}$
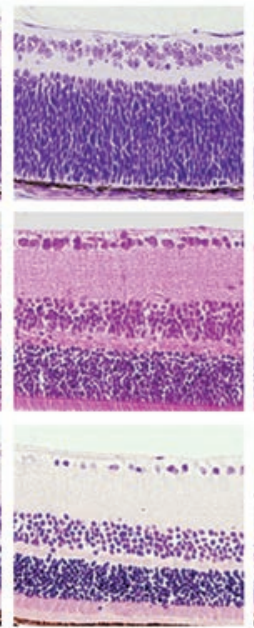

B
EAAC1+/-
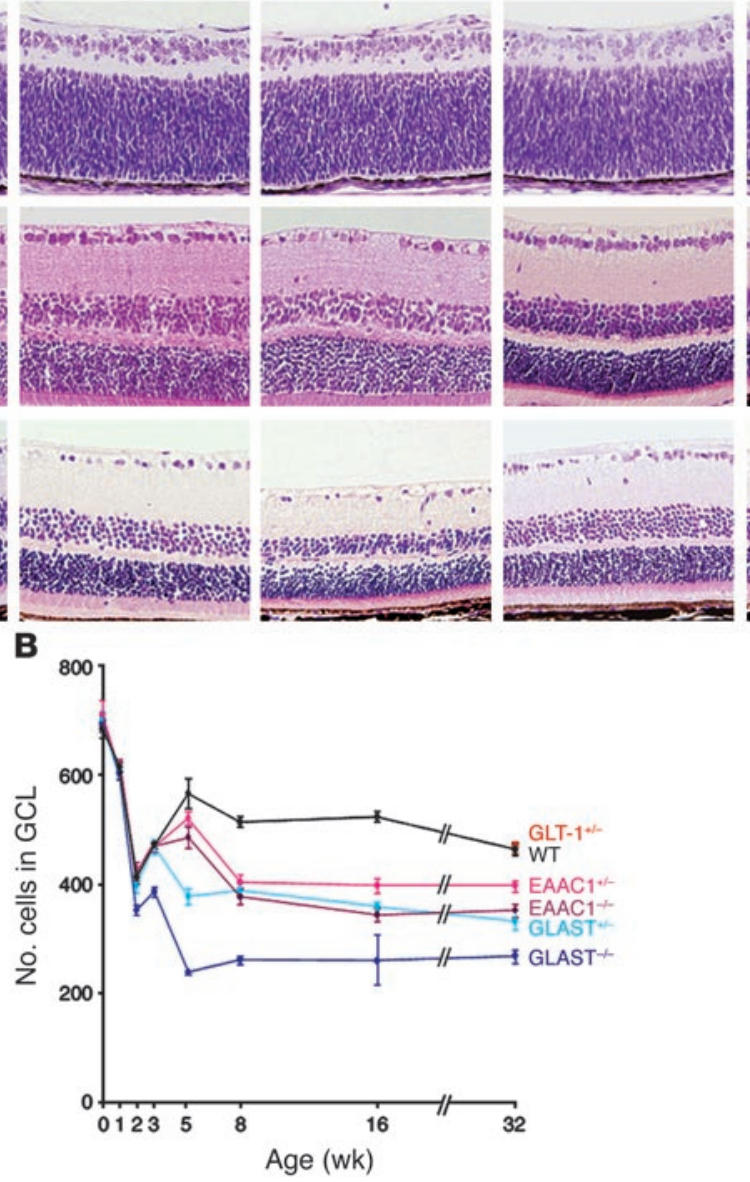

$\mathrm{EAAC1}^{-1-}$

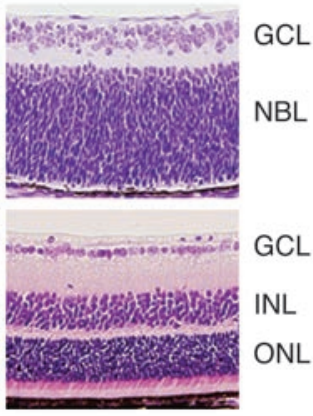

NBL

GCL INL ONL 


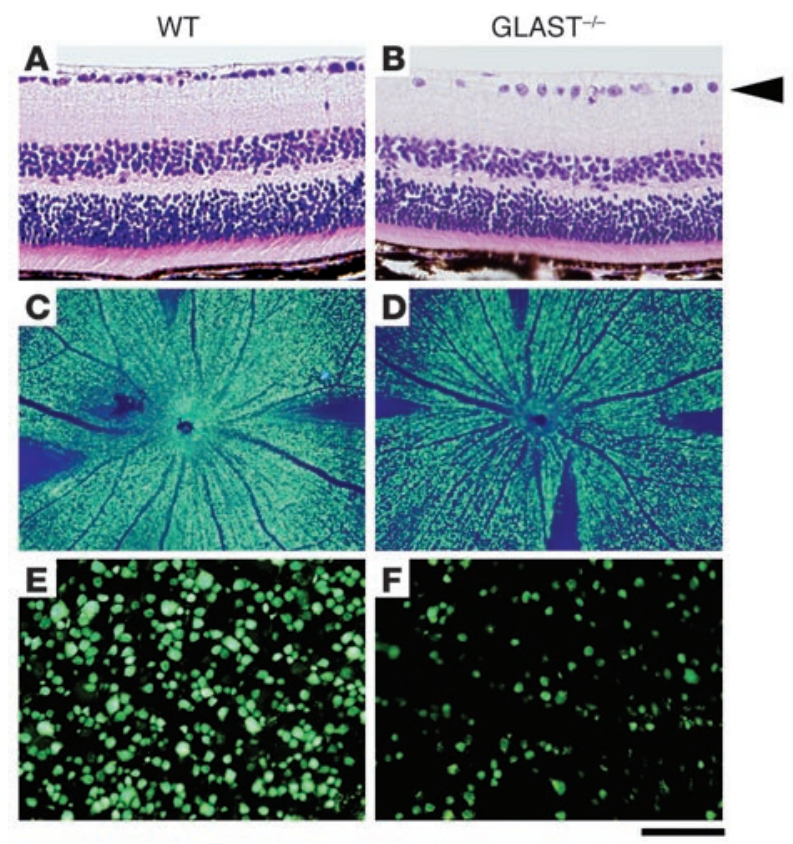

which cell number was decreased in the ganglion cell layer (GCL) $(53 \% \pm 6 \% ; n=6, P<0.0001)$ and the inner nuclear layer (INL) $(81 \% \pm 11 \% ; n=6, P<0.05)$ but not in the ONL $(97 \% \pm 5 \% ; n=6$, $P=0.34)$. As nearly half of the cells in the rodent GCL are displaced amacrine cells, we distinguished RGCs from displaced amacrine cells by retrograde labeling (16). The number of RGCs per $\mathrm{mm}^{2}$ in retinae of 8-month-old GLAST ${ }^{-/}$mice $(2022 \pm 48 ; n=18)$ was significantly reduced compared with that in WT mice (4011 \pm 106 ; $n=18, P<0.0001$ ) (Figure 3, C-F). We also examined RGC number by counting the cells located in the GCL. Because quantification of RGC loss by GCL cell count $(49 \% \pm 2 \% ; n=6)$ was consistent with that by RGC labeling $(50 \% \pm 1 \% ; n=18, P=0.89)$ in 8 -monthold GLAST ${ }^{-/-}$mice, we examined the GCL cell number from P0 to 8 months in all mutant mice (Figure 2B). Significant RGC loss was also observed in $\mathrm{GLAST}^{-/-}$mice after 2 weeks of age, $\mathrm{GLAST}^{+/-}$mice after 5 weeks, and $\mathrm{EAAC}^{+/-}$and $\mathrm{EAAC1} 1^{-/-}$mice after 8 weeks. We were unable to examine 8 -month-old GLT-1/- mice (17) because most of them died within 3 weeks; however, RGC number in 8-month-old GLT-1 ${ }^{+/-}$mice was normal compared with that in WT mice $(101 \% \pm 2 \% ; n=10$; Figure $2 \mathrm{~B}$ and Figure 4$)$. Thus, GLT-1 mutant mice were not evaluated further.

Degeneration of optic nerve in GLAST- and EAAC1-deficient mice. Degeneration of the optic nerve is one of the hallmarks of glaucoma. Consistent with severe RGC loss, optic nerve cupping (Figure 5B) and thinning of the optic nerve (Figure 5D) were apparent in 8-month-old GLAST ${ }^{-/-}$mutants. Quantitative analysis revealed that optic nerve area in GLAST ${ }^{-/-}$mice $\left(0.074 \pm 0.002 \mathrm{~mm}^{2}\right.$; $n=6)$ was significantly decreased compared with that in WT mice $\left(0.096 \pm 0.005 \mathrm{~mm}^{2} ; n=6, P=0.0019\right)$. To analyze morphological changes in the optic nerve, semithin transverse sections were cut and stained with toluidine blue. The degenerating axons in 5-week-old GLAST ${ }^{-/-}$mutants had abnormally dark axonal profiles (Figure 5F). In addition, compared with WT mice (Figure 5E), the density of axons through the optic nerve was clearly declined in GLAST $^{-/-}$mice (Figure 5F). We next examined the iridocorneal angle in GLAST $^{-/-}$mutants and found that it was well formed

\section{Figure 3}

RGC degeneration in GLAST ${ }^{-/-}$mice. H\&E-stained sections show a decreased number of cells in the GCL in GLAST-1- mouse (arrowhead) (A and B). Retrogradely labeled RGCs in GLAST ${ }^{-1-}$ mouse were decreased compared with those in WT mouse (C and D). $\mathbf{E}$ and $\mathbf{F}$ are magnified images of $\mathbf{C}$ and $\mathbf{D}$, respectively. Scale bar: $100 \mu \mathrm{m}$ (A, B, $\mathbf{E}$, and F); $500 \mu \mathrm{m}$ (C and D).

with an obvious Schlemm canal and a normal extent of trabecular meshwork (Figure $5 \mathrm{H}$ ). EAAC1 ${ }^{-/-}$mutants also showed excavation of the optic nerve and normal iridocorneal angle morphology (data not shown).

IOP measurement in GLAST- and EAAC1-deficient mice. GLAST and EAAC1 mutant mice showed POAG-like phenotype, which suggests the involvement of increased IOP. Therefore, we examined the IOPs of WT, EAAC1 $1^{-/}$, and GLAST ${ }^{-/-}$mice. IOP measurement was carried out for both young ( 4 week) and adult ( 9 to 11 month) mice around $9 \mathrm{pm}$, when IOP is highest in mouse eyes (18). The IOPs of EAAC1 $1^{-/-}$and GLAST $^{-/-}$mice were not significantly increased compared with WT at both time points (Figure 5I). These results suggest that RGC loss and glaucomatous-like changes of the optic nerve in these mutants are IOP-independent.

Impaired visual function in GLAST-deficient mice. To determine whether the observed degeneration of RGCs and the optic nerve is functionally significant, we analyzed multifocal electroretinograms (mfERGs) of GLAST ${ }^{-/}$mouse retina. RGCs contribute to the human mfERG response, and the second-order kernel (2K), which appears to be a sensitive indicator of inner retinal dysfunction (19), is impaired in glaucoma patients (20). The averaged $2 \mathrm{~K}$ responses of 10 mice are shown in Figure 6A. The response topography demonstrated that the $2 \mathrm{~K}$ component derived from GLAST ${ }^{-/-}$ mice tended to be shorter in all visual fields compared with that of WT mice (Figure 6B). Quantitative analysis revealed that the $2 \mathrm{~K}$ amplitude was reduced by about half in $\mathrm{GLAST}^{-/}$mice compared with that in WT mice $(P<0.005)$ (Figure 6C), suggesting that RGC loss is functionally significant in $\mathrm{GLAST}^{-/}$mice.

Role of glutamate neurotoxicity in RGC degeneration in GLAST-deficient mice. To determine the contribution of excitotoxicity to RGC death in these models, we first measured the intravitreal glutamate concentration in WT and mutant mice. Glutamate concentration was not significantly increased in GLAST or EAAC1 mutant mice (Figure 7A). Because an increase in glutamate concentration in the extracellular space just adjacent to RGCs might be obscured due to dilution within the vitreous (21), failure to detect glutamate elevation in the vitreous fluid does not necessarily exclude glutamate involvement in RGC degeneration in the mutants. To assess glu-

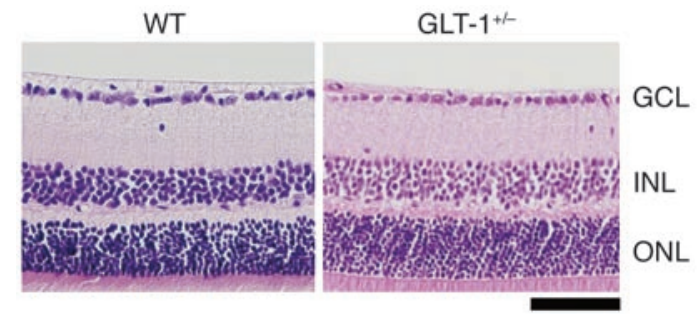

\section{Figure 4}

Normal retinal structure in GLT-1+/- mouse. H\&E-stained sections show the absence of RGC degeneration in an 8-month-old GLT-1+/mouse. Scale bar: $50 \mu \mathrm{m}$. 

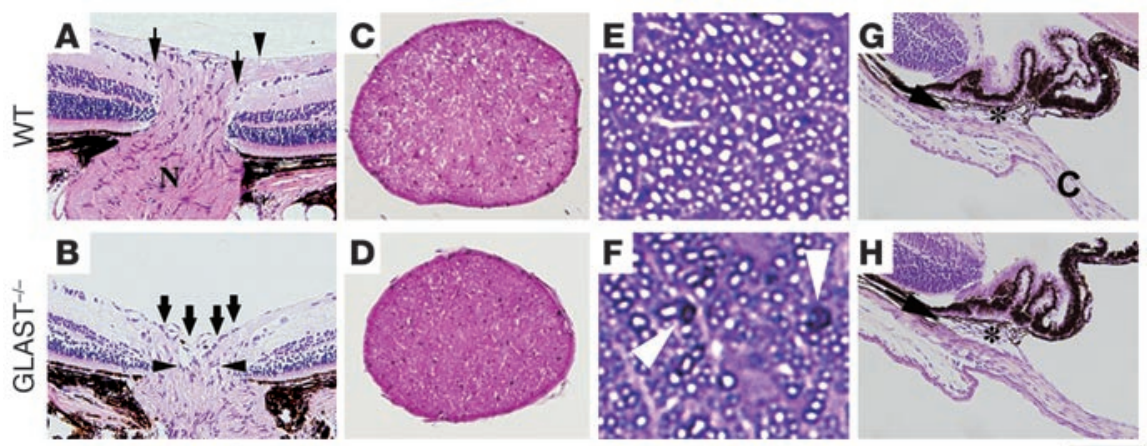

I

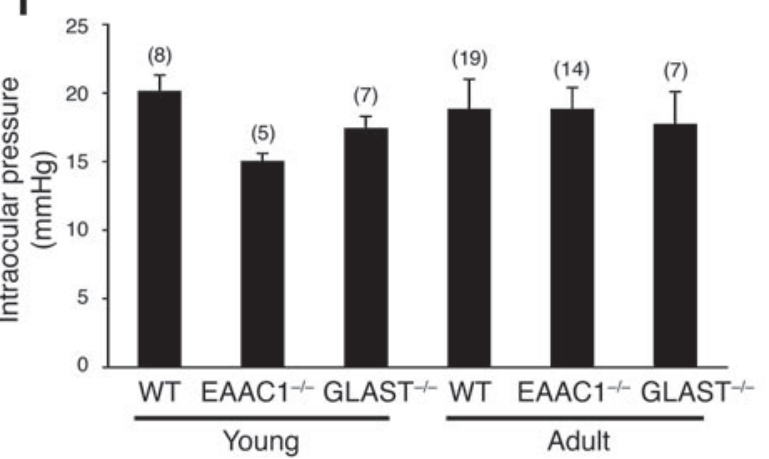

\section{Figure 5}

Optic nerve degeneration and normal IOP in glutamate transporter mutant mice. (A and B) Optic nerve atrophy in 8-month-old GLAST-1- mice. In WT mice (A), the nasal and temporal nerve fibers (arrows) are beneath the internal limiting membrane (arrowhead) and enter a well-formed optic nerve (N). In GLAST ${ }^{-/-}$mice (B), the nerve fiber layer has become thin and almost absent as it enters the nerve (bold arrows). Cupping extends to the posterior aspect of the inner retinal layer (arrowheads). (C and D) Thinning of optic nerve is apparent in GLAST-/- (D) compared with WT (C) mice. (E and $\mathbf{F}$ ) Staining of semithin sections with toluidine blue reveals the presence of abnormally dark axonal profiles (arrowheads) and decline of axons in GLAST ${ }^{-/-}(\mathbf{F})$ compared with WT $(\mathbf{E})$ mice. (G and $\left.\mathbf{H}\right)$ Aqueous humor drainage structures in WT $(\mathbf{G})$ and $\mathrm{GLAST}{ }^{-/-}(\mathbf{H}) \mathrm{mice}^{-}$ Iridocorneal angle in $\mathrm{GLAST}^{-/-}$mice is normal with an obvious Schlemm's canal (arrow) and trabecular meshwork (asterisk) compared with those in WT mice. The angle recess between the cornea (C) and iris is wide open. (I) IOP of young (4 weeks old) and adult (9-11 months old) mice. Sample numbers are indicated in parentheses. Scale bar: $200 \mu \mathrm{m}(\mathbf{A}, \mathbf{B}, \mathbf{G}$, and $\mathbf{H}) ; 130 \mu \mathrm{m}(\mathbf{C}$ and $\mathbf{D}) ; 9 \mu \mathrm{m}(\mathbf{E}$ and $\mathbf{F})$.

tamate involvement in RGC degeneration, we next examined the effect of the NMDA receptor antagonist memantine (22) on RGC degeneration in GLAST mutants. Memantine $(10 \mathrm{mg} / \mathrm{kg}$ injected daily) prevented RGC loss when applied from P7 to P13 (Figure 7B), and the RGC number in GLAST ${ }^{-/}$mice was normal at P14 (Figure 7C). The protective effect of glutamate receptor antagonist suggests that glutamate neurotoxicity is partly involved in RGC loss in GLAST mutant mice. However, since glutamate concentration in the vitreous fluid was normal, other factors, in addition to glutamate neurotoxicity, might contribute to RGC degeneration of GLAST-deficient mice.

Role of oxidative stress in RGC degeneration of GLAST- and EAAC1deficient mice. In addition to excitotoxicity, oxidative stress has also been proposed to contribute to RGC death in glaucoma $(23,24)$. In adult $\mathrm{GLAST}^{-/-}$and EAAC1 $1^{-/-}$mice, lipid hydroperoxides were increased in retinae, suggesting the involvement of oxidative stress in RGC loss (Figure 8A). Previous studies demonstrated that glutathione, a tripeptide of glutamate, cysteine, and glycine, has a central role in protecting RGCs against oxidative stress and that glutamate uptake is a rate-limiting step in glial glutathione synthesis $(25,26)$. Because retinal glutathione was specifically distributed in Müller cells (Figure 8B), we hypothesized that oxidative stress may result from impaired glial glutathione synthesis in $\mathrm{GLAST}^{-/-}$mice. In agreement with this hypothesis, glutathione content in the retina and in cultured Müller cells was decreased in GLAST $^{-/-}$mice (Figure 8C). A similar reduction in glutathione level was recently reported in the plasma of human POAG patients (27). To confirm that the reduction in Müller cell glutathione levels makes RGCs more vulnerable to oxidative stress, we examined the effect of $\mathrm{H}_{2} \mathrm{O}_{2}$ on WT RGCs in a mixed culture with Müller cells from GLAST ${ }^{-/-}$or WT mice. We used a concentration of $\mathrm{H}_{2} \mathrm{O}_{2}$ that does not induce Müller cell death. RGC loss was significantly higher when RGCs were mixed with Müller cells from GLAST ${ }^{-/-}$mice than when mixed with Müller cells from WT mice (Figure 8D). These results indicate that GLAST deficiency leads to glaucomatous-like RGC loss in 2 ways: first, by excessive stimulation of NMDA receptors and second, by reducing the glutathione levels in Müller cells, thus making RGCs more vulnerable to oxidative stress.

Unlike glial GLAST, neuronal EAAC1 does not play a major role in clearing glutamate from the extracellular space $(15,28)$. Instead, EAAC1 can transport cysteine, an obligate precursor for neuronal glutathione synthesis, far more effectively than GLAST (29). Aoyama et al. recently reported that EAAC1 deficiency leads to impaired neuronal glutathione metabolism and age-dependent brain atrophy (29). In EAAC1 1 - mutant mice, retinal glutathione content was not decreased (Figure 8C). These measurements represent bulk glutathione content in retinal homogenates from 
A
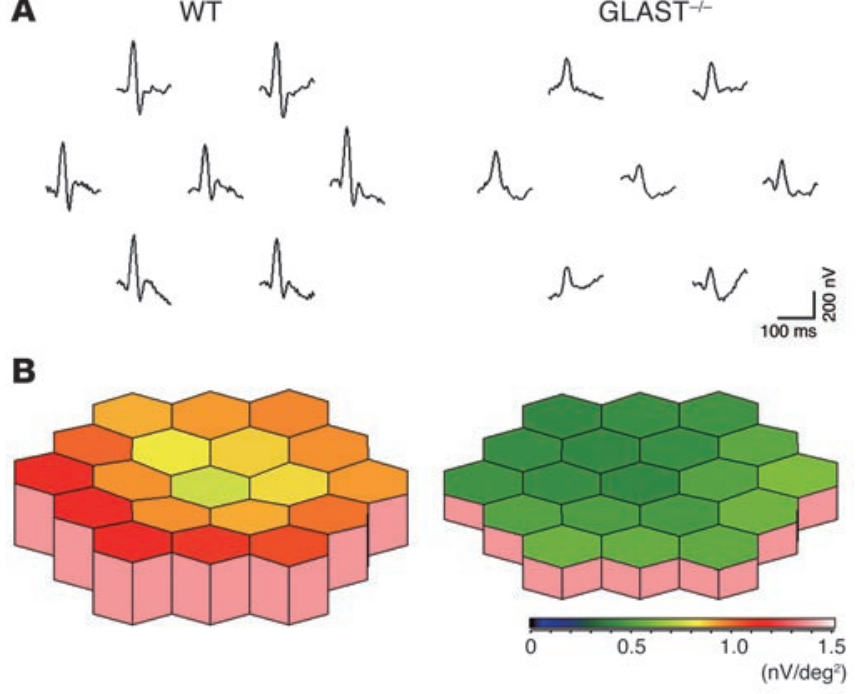

C

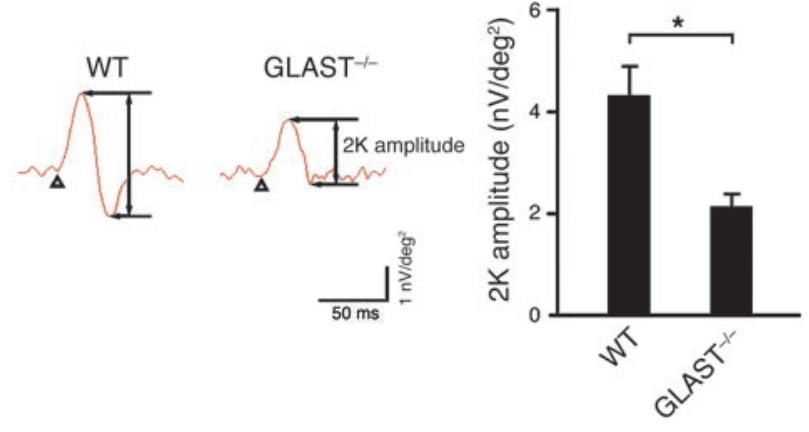

EAAC1 ${ }^{-/-}$and WT mice. Thus, glutathione deficiency in RGCs of EAAC $1^{-/}$mice may not be apparent because a large share of retinal glutathione is localized to Müller cells (26), which do not express EAAC1. To directly determine the vulnerability of EAAC1-/RGCs to oxidants, primary cultures of mutant and WT RGCs were treated with $\mathrm{H}_{2} \mathrm{O}_{2}$. Cultured RGCs from EAAC1 $1^{-/}$mice were susceptible to $\mathrm{H}_{2} \mathrm{O}_{2}$ compared with those from WT mice (Figure 8E). These findings suggest that EAAC1 deficiency makes RGCs more vulnerable to oxidative stress.

\section{Discussion}

Here, we show that GLAST- and EAAC1-knockout mice show progressive RGC loss and glaucomatous optic nerve degeneration without elevated IOP. Our study raises the possibility that dysfunction of the glutamate transporters GLAST (EAAT1 in humans) and EAAC1 (EAAT3 in humans) plays a role in RGC death in human NTG. It is reported that EAAT1 is downregulated in human glaucoma (30). In addition, a decrease of EAAT1 has been shown in fibroblasts from patients with Alzheimer disease (31). Considering the high frequency of POAG in Alzheimer disease patients (32), common mechanisms, such as GLAST dysfunction, might contribute to the 2 diseases. These findings indicate that GLAST- and EAAC1-knockout mice are useful as models of NTG.

Because GLAST is a major glutamate transporter in the mammalian retina, loss of GLAST is expected to render the retina highly susceptible to excitotoxic damage. However, we previously reported that the $\mathrm{GLAST}^{-/-}$retina showed no signs of RGC degeneration although it was more sensitive to prolonged ischemia than normal

\section{Figure 6}

Impaired multifocal electroretinogram in GLAST ${ }^{-/}$mice. (A) Averaged responses of $2 \mathrm{~K}$ from 10 mice. The visual stimulus was applied to 7 different areas in the retina. The 7 individual traces demonstrate the average responses to the visual stimulus at the corresponding stimulus area. (B) Three-dimensional plots showing the amplitude variation across the arrays in A. (C) Quantitative analysis of $2 \mathrm{~K}$ amplitude. The response amplitudes for each stimulus element were added and the result was divided by the total area of the visual stimulus. $n=10$ per group; ${ }^{*} P<0.005$. Values in $\mathbf{B}$ and $\mathbf{C}$ are given in $\mathrm{nV}$ per square degree $\left(\mathrm{nV} / \mathrm{deg}^{2}\right)$.

retina (13). In the present study, GLAST ${ }^{-/-}$mice showed spontaneous RGC loss and glaucomatous optic nerve degeneration. What factors account for this discrepancy? One possibility is the genetic background of the mice used. In previous studies, we used GLAST ${ }^{-/-}$ mice on a mixed C57BL/ $6 \times 129$ genetic background. During the course of backcrossing to a C57BL/ 6 strain, GLAST ${ }^{-/-}$mice showed spontaneous NTG-like RGC degeneration. This finding can allow us to identify strain-specific modifier genes that might suppress or enhance RGC degeneration. It is intriguing that the C57BL/6J mice with mutations in the optineurin (OPTN) gene, which have been associated with human POAG and NTG, show extensive RGC loss (33). Thus, the C57BL/6J strain may have a potentially pathogenic allele of Optn. Although more studies are needed to determine whether the NTG-like phenotype in $\mathrm{GLAST}^{-/-}$mice on a C57BL/6J strain is related to Optn, such studies will identify new candidate molecules/pathways that may contribute to RGC survival.

The results of the present study suggest that glial GLAST and neuronal EAAC1 play differential roles in preventing RGC degeneration. GLAST is essential not only to keep the extracellular glutamate concentration below the neurotoxic level, but also to maintain the glutathione levels in Müller cells by transporting glutamate, the substrate for glutathione synthesis, into the cells. In contrast, the main role of EAAC1 is to transport cysteine into RGCs as a precursor for neuronal glutathione synthesis. Thus, GLAST deficiency leads to RGC degeneration caused by both excitotoxicity and oxidative stress whereas EAAC1 deficiency induces RGC loss mainly through oxidative stress. In the present paper, we demonstrate that the availability of glutamate is limiting for the maintenance of normal intracellular glutathione level in Müller cells. This observation differs from findings in other tissues, where cysteine was identified as the rate-limiting substance $(34,35)$. How can this be explained? Previous studies demonstrated that Müller cells possess a very fast glutamate turnover (36). A major part of the available intracellular glutamate is used for glutamine synthesis catalyzed by the enzyme GS, which is exclusively located in Müller cells (37). This makes the intracellular glutamate concentration of Müller cells strongly dependent on glutamate uptake via the glutamate transporter GLAST. In contrast, the cysteine turnover of Müller cells seems to be rather slow (36). Therefore, the rate-limiting factor for glutathione levels in Müller cells is not the cystine supply to the cells but rather the provision of glutamate.

Glutamate excitotoxicity and oxidative stress have been proposed to contribute to retinal damage in various eye diseases, including retinal ischemia, glaucoma, diabetic retinopathy, and age-related macular degeneration (38-40). Therefore, the design of compounds capable of activating glutamate uptake by GLAST represents a novel strategy for the management of glaucoma and various forms of retinopathy $(41,42)$. We also showed that RGCs 
A

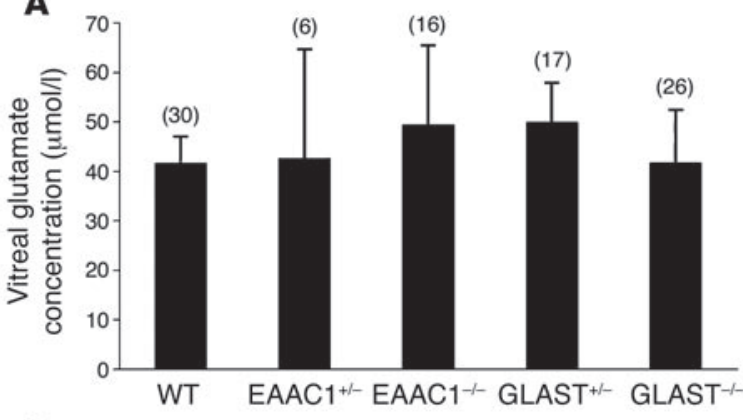

B
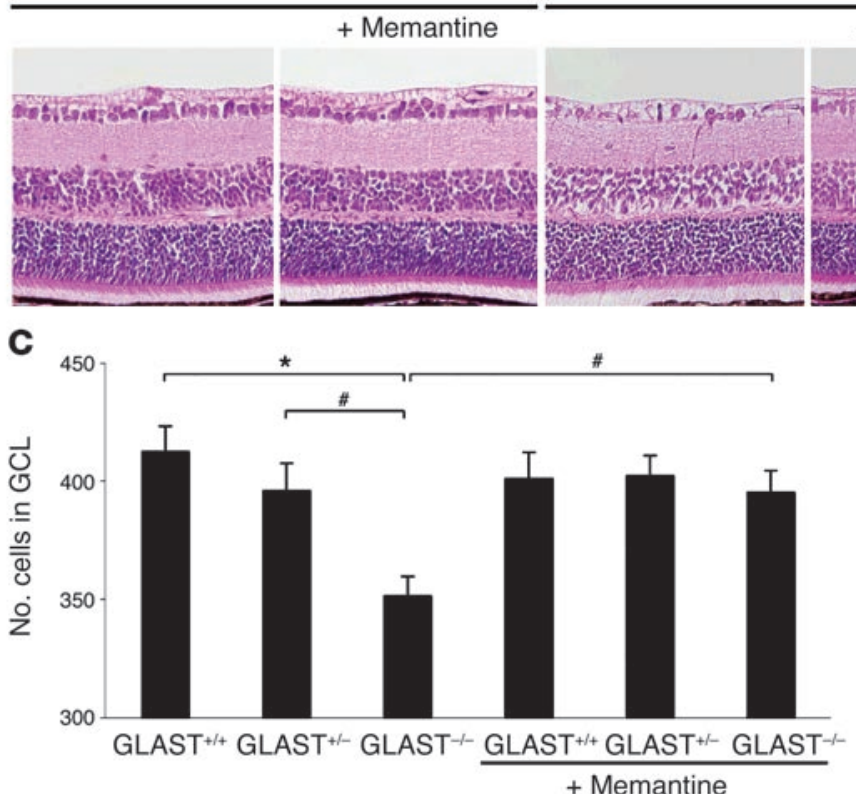

\section{Figure 7}

Effect of glutamate neurotoxicity on RGC degeneration. (A) Intravitreal glutamate concentration in glutamate transporter mutant mice. Sample numbers are indicated in parentheses. (B) H\&Estained P14 retinal sections from WT and GLAST ${ }^{-/}$ mice with or without treatment with memantine (10 mg/kg, i.p.) daily from P7 to P13. (C) Quantitative analysis of RGC number following memantine administration. The number of neurons in the GCL was counted in the retinal section from one ora serrata through the optic nerve to the other ora serrata. $n=6$ per group. ${ }^{\#} P<0.05 ;{ }^{*} P<0.005$. Scale bar: $50 \mu \mathrm{m}(\mathbf{B})$. cocultured with Müller cells from GLAST ${ }^{-/}$mice are susceptible to free radical stimulation (Figure 8D). We previously reported that neurotrophins alter the production of some trophic factors in Müller cells, which indirectly leads to neural cell survival during photoreceptor degeneration $(43,44)$. We suggest that such a glia-neuron network is functional in various forms of neurodegenerative diseases, and the messengers between glia and neurons may be different according to the situation. In addition, each single Müller cell extends from the outer to the inner surface of the retina. This allows the Müller cells to form elaborate intimate contacts with the somata of all types of retinal neurons as well as with the fibers and synapses in the neuropile of the 2 plexiform layers. Their ubiquitous presence makes the Müller cells a suitable target for drug delivery and gene therapy in retinal degenerative diseases. Furthermore, recent studies have shown that Müller cells could proliferate after neurotoxic damage and produce bipolar cells and rod photoreceptors in the adult mammalian retina $(45,46)$. Therefore, Müller cells may be a new therapeutic target for both neuroprotection and regeneration in retinal degenerative diseases $(47,48)$.

\section{Methods}

Mice. Experiments were performed using EAAC1 ${ }^{+/-}$, EAAC1/- (15), GLT-1+/- (17), GLAST +/-, and GLAST ${ }^{-/-}$(14) mice with approval from the Institutional Animal Care and Use Committee of Tokyo Medical and Dental University and the Tokyo Metropolitan Institute for Neuroscience. All the mice used in this study were backcrossed with C57BL/6 more than 8 times.

Immunohistochemistry. Frozen $12-\mu \mathrm{m}$-thick retinal sections were incubated with 1 of 4 sets of primary antibody mix. Primary antibodies used were mouse anti-GS (Chemicon International) and rabbit anti-GLAST (13) or anti-glutathione (Signature Immunologics); and mouse anti-calretinin (Chemicon International) and rabbit anti-EAAC1 (49) or anti-GLT-1 (13). Fluorescence immunohistochemistry was performed using Cy2-conjugated donkey anti-mouse IgG and Cy3-conjugated donkey anti-rabbit IgG (Jackson ImmunoResearch Laboratories Inc.). Sections were examined by fluorescence microscopy (Olympus).

Histological and morphometric studies. Paraffin sections (7- $\mu$ m thick) of retinal specimens were cut through the optic nerve and stained with $\mathrm{H} \& \mathrm{E}$. RGC number and the extent of retinal degeneration were quantified in 3 ways. First, the cell density of each layer was analyzed (50). Second, in the same sections, the number of neurons in the GCL was counted from one ora serrata through the optic nerve to the other ora serrata. Third, RGCs were retrogradely labeled from the superior colliculus with Fluoro-Gold (Fluorochrome) (16). Seven days after Fluoro-Gold application, eyes were enucleated and retinas were detached and prepared as flattened whole mounts in $4 \%$ paraformaldehyde in $0.1 \mathrm{M} \mathrm{PBS}$ solution. GCL was examined in wholemounted retinae with fluorescence microscopy to determine the RGC density. Four standard areas $\left(0.04 \mathrm{~mm}^{2}\right)$ of each retina at the point of $0.1 \mathrm{~mm}$ from the optic disc were randomly chosen, labeled cells were counted by observers 


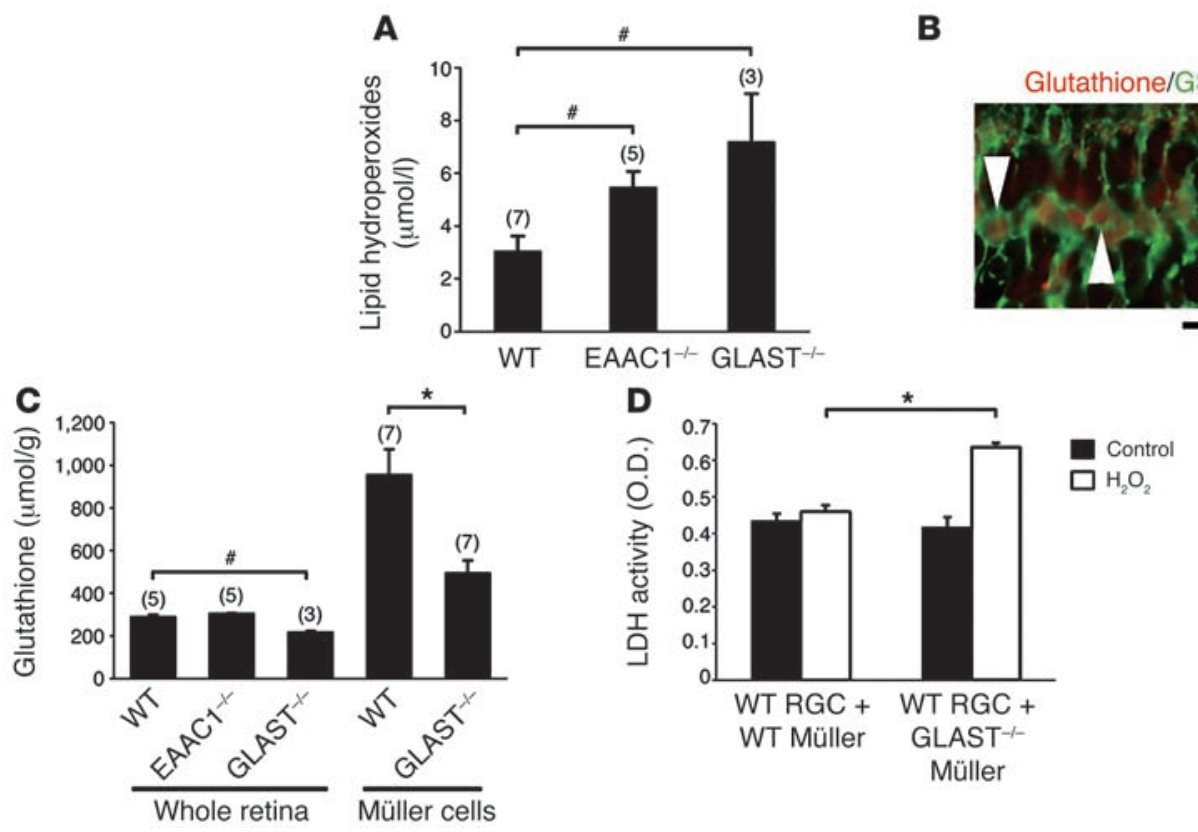

B

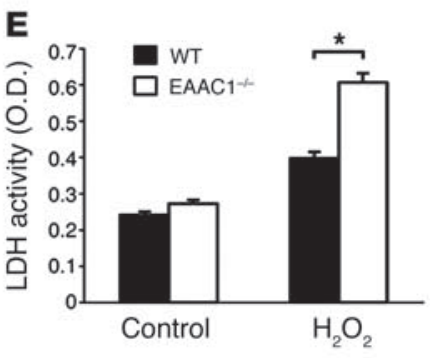

Figure 8

Increased oxidative stress in glutamate transporter mutant mice. (A) Lipid hydroperoxide concentration in whole retina of WT, EAAC1 ${ }^{-1-}$, and GLAST ${ }^{-/}$mice. Sample numbers are indicated in parentheses. (B) Immunohistochemical analysis of mouse retina double-stained with glutathione and GS. Glutathione-like immunoreactivity was observed in Müller glial cells (arrowheads). Scale bar: $20 \mu \mathrm{m}$. (C) Mean glutathione concentration in whole retina and cultured Müller cells. Sample numbers are indicated in parentheses. (D) Lactate dehydrogenase (LDH) release from $\mathrm{H}_{2} \mathrm{O}_{2}$-treated RGCs cocultured with WT or GLAST-1- Müller cells. $n=3$ per group. (E) Lactate dehydrogenase release from $\mathrm{H}_{2} \mathrm{O}_{2}$-treated RGCs from WT or EAAC1 ${ }^{-/-}$mice. $n=4$ per group. ${ }^{\sharp} P<0.05$; ${ }^{*} P<0.005$.

blinded to the identity of the mice, and the average number of RGCs $/ \mathrm{mm}^{2}$ was calculated. The changes in RGC number were expressed as percentages of the WT control eyes. In some experiments, memantine $(10 \mathrm{mg} / \mathrm{kg}$; Merz) was injected i.p. into GLAST ${ }^{-/}$mice and their littermates daily from P7 to P13. These mice were sacrificed on P14 and processed for RGC count.

For the analysis of the optic nerve, frozen $10-\mu \mathrm{m}$-thick sections were cut at $2.5 \mathrm{~mm}$ from the eyeball and stained with $\mathrm{H} \& \mathrm{E}$. Quantitative analysis of the optic nerve area was carried out using a computerized image analysis program (Scion Image Beta 4.0.3; Scion Corporation). For detailed morphological analysis, optic nerves were fixed in $2 \%$ glutaraldehyde and $2 \%$ paraformaldehyde in $0.1 \mathrm{M}$ phosphate buffer overnight at $4^{\circ} \mathrm{C}$. After dissection, the pieces of tissue were placed in $1 \%$ osmium tetroxide. After dehydration, the pieces were embedded in EPON (Nisshin EM). Transversal semithin (1 $\mu \mathrm{m})$ sections were stained with $0.2 \%$ toluidine blue in $1.0 \%$ sodium borate.

IOP measurement. IOP was directly measured by a microneedle method in anesthetized mice as described previously $(18,51)$. The microneedle was connected to a pressure transducer, which relayed its signal to a bridge amplifier and then to an analog-to-digital converter and a computer (World Precision Instruments). The microneedle tip was inserted through the cornea and the data were automatically collected online into a computer database. To minimize variation, data were collected during a time window 4-6 minutes after injection of anesthetic, during which time IOP plateaued (51). Animal age was 4 weeks or 9-11 months, and body weight ranges were $13-24 \mathrm{~g}$ or $28-36 \mathrm{~g}$ at the time of IOP measurement, respectively. Since 24-hour IOP pattern in mouse eyes is biphasic and IOP is highest around 9:00 pm (18), we examined IOP between 8:00 pm and 11:00 pm.

mfERGs. Mice (12-16 weeks old) were anesthetized by i.p. injection of a mixture of xylazine $(10 \mathrm{mg} / \mathrm{kg})$ and ketamine $(25 \mathrm{mg} / \mathrm{kg})$. Pupils were dilat- ed with $0.5 \%$ phenylephrine hydrochloride and $0.5 \%$ tropicamide. mfERGs were recorded using a VERIS 5.1 system (Electro-Diagnostic Imaging Inc.). The visual stimulus consisted of 7 hexagonal areas scaled with eccentricity. The stimulus array was displayed on a high-resolution black and white monitor driven at a frame rate of $100 \mathrm{~Hz}$. The $2 \mathrm{~K}$, which is impaired in patients with glaucoma (20), was analyzed.

Glutamate assay. Vitreous samples $(5-10 \mu \mathrm{l})$ were surgically extracted from adult mice, and glutamate was analyzed by serial enzymatic reactions as previously reported (52).

Cell culture. RGCs (16) and Müller cells (44) were prepared from P7 mice. In some experiments, WT RGCs were cocultured with Müller cells from GLAST $^{-/-}$mice or their WT littermates. These culture cells were stimulated with $200 \mu \mathrm{M} \mathrm{H}_{2} \mathrm{O}_{2}$ for 16 hours, and RGC death rate was analyzed using a lactate dehydrogenase cytotoxic test kit (Wako) as previously reported (16).

Lipid peroxidation and glutathione assay. The concentrations of lipid peroxides and glutathione were measured using Bioxytech LPO-560 (Oxis Health Products Inc.) and Glutathione assay kit (Cayman Chemical) per the manufacturers' protocols.

Statistics. For statistical comparison of 2 samples, we used a 2-tailed Student's $t$ test or Mann-Whitney $U$ test. In other cases, we also used 1-factor ANOVA and Tukey-Kramer test. Data are presented as the mean \pm SEM. $P<0.05$ was regarded as statistically significant.

\section{Acknowledgments}

We thank X. Guo, K. Ajiki, and M. Ichikawa for technical assistance. This study was supported in part by grants from the Ministry of Education, Culture, Sports, Science and Technology of Japan, the Tokyo Biochemical Research Foundation, the Naito 
Foundation, and the Uehara Memorial Foundation (to T. Harada). C. Harada was supported by a fellowship from Japan Society for the Promotion of Science for Young Scientists.

Received for publication August 28, 2006, and accepted in revised form April 24, 2007.

1. Quigley, H.A. 1996. Number of people with glaucoma worldwide. Br. J. Ophthalmol. 80:389-393.

2. Weinreb, R.N., and Khaw, P.T. 2004. Primary openangle glaucoma. Lancet. 363:1711-1720.

3. Klein, B.E., et al. 1992. Prevalence of glaucoma. The Beaver Dam Eye Study. Ophthalmology. 99:1499-1504.

4. Bonomi, L., et al. 1998. Prevalence of glaucoma and intraocular pressure distribution in a defined population. The Egna-Neumarkt Study. Ophthalmology. 105:209-215.

5. Iwase, A., et al. 2004. The prevalence of primary open-angle glaucoma in Japanese: the Tajimi Study. Ophthalmology. 111:1641-1648.

6. Anderson, D.R. 2003. Collaborative normal tension glaucoma study. Curr. Opin. Ophthalmol. 14:86-90.

7. Wiggs, J.L. 2007. Genetic etiologies of glaucoma. Arch. Ophthalmol. 125:30-37.

8. Osborne, N.N., Lascaratos, G., Bron, A.J., Chidlow, G., and Wood, J.P. 2006. A hypothesis to suggest that light is a risk factor in glaucoma and the mitochondrial optic neuropathies. Br. J. Ophthalmol. 90:237-241.

9. Lindsey, J.D., and Weinreb, R.N. 2005. Elevated intraocular pressure and transgenic applications in the mouse. J. Glancoma. 14:318-320.

10. Danbolt, N.C. 2001. Glutamate uptake. Prog. Neurobiol. 65:1-105.

11. Rauen, T. 2000. Diversity of glutamate transporter expression and function in the mammalian retina. Amino Acids. 19:53-62.

12. Dalton, R. 2001. Private investigations. Nature. 411:129-130.

13. Harada, T., et al. 1998. Functions of the two glutamate transporters GLAST and GLT-1 in the retina. Proc. Natl. Acad. Sci. U. S. A. 95:4663-4666.

14. Watase, K., et al. 1998. Motor discoordination and increased susceptibility to cerebellar injury in GLAST mutant mice. Eur. J. Neurosci. 10:976-988.

15. Peghini, P., Janzen, J., and Stoffel, W. 1997. Glutamate transporter EAAC-1-deficient mice develop dicarboxylic aminoaciduria and behavioral abnormalities but no neurodegeneration. EMBOJ. 16:3822-3832.

16. Harada, C., et al. 2006. Role of apoptosis signalregulating kinase 1 in stress-induced neural cell apoptosis in vivo. Am. J. Pathol. 168:261-269.

17. Tanaka, K., et al. 1997. Epilepsy and exacerbation of brain injury in mice lacking the glutamate transporter GLT-1. Science. 276:1699-1702.

18. Aihara, M., Lindsey, J.D., and Weinreb, R.N. 2003. Twenty-four-hour pattern of mouse intraocular pressure. Exp. Eye Res. 77:681-686.

19. Hasegawa, S., Ohshima, A., Hayakawa, Y., Takagi, M., and Abe, H. 2001. Multifocal electroretinograms in patients with branch retinal artery occlusion. Invest. Ophthalmol. Vis. Sci. 42:298-304.

20. Sutter, E.E., and Bearse, M.A., Jr. 1999. The optic
Address correspondence to: Kohichi Tanaka, Laboratory of Molecular Neuroscience, School of Biomedical Science and Medical Research Institute, Tokyo Medical and Dental University, 1-5-45 Yushima, Bunkyo-ku, Tokyo 113-8510, Japan. Phone: 81-3-5803-5846; Fax: 81-3-5803-5843; E-mail: tanaka. aud@mri.tmd.ac.jp. nerve head component of the human ERG. Vision Res. 39:419-436.

21. Adachi, K., et al. 1998. Mechanisms of the pathogenesis of glutamate neurotoxicity in retinal ischemia. Graefes Arch. Clin. Exp. Ophthalmol. 236:766-774.

22. Lipton, S.A. 2004. Paradigm shift in NMDA receptor antagonist drug development: molecular mechanism of uncompetitive inhibition by memantine in the treatment of Alzheimer's disease and other neurologic disorders. J. Alzheimers Dis. 6(6 Suppl.):S61-S74.

23. Klein, J.A., et al. 2002. The harlequin mouse mutation downregulates apoptosis-inducing factor. Nature. 419:367-374.

24. Tezel, G. 2006. Oxidative stress in glaucomatous neurodegeneration: mechanisms and consequences. Prog. Retin. Eye Res. 25:490-513.

25. Schulz, J.B., Lindenau, J., Seyfried, J., and Dichgans, J. 2000. Glutathione, oxidative stress and neurodegeneration. Eur. J. Biochem. 267:4904-4911.

26. Huster, D., Reichenbach, A., and Reichelt, W. 2000. The glutathione content of retinal Müller (glial) cells: effect of pathological conditions. Neurochem. Int. 36:461-469.

27. Gherghel, D., Griffiths, H.R., Hilton, E.J., Cunliffe, I.A., and Hosking, S.L. 2005. Systemic reduction in glutathione levels occurs in patients with primary open-angle glaucoma. Invest. Ophthalmol. Vis. Sci. 46:877-883.

28. Sarthy, V.P., et al. 2005. Glutamate transporter in retinal Müller cells from glutamate/aspartate transporter (GLAST) knockout mice. Glia. 49:184-196.

29. Aoyama, K., et al. 2006. Neuronal glutathione deficiency and age-dependent neurodegeneration in the EAAC1 deficient mouse. Nat. Neurosci. 9:119-126.

30. Naskar, R., Vorwerk, C.K., and Dreyer, E.B. 2000. Concurrent downregulation of a glutamate transporter and receptor in glaucoma. Invest. Ophthalmol. Vis. Sci. 41:1940-1944.

31. Zoia, C.P., et al. 2005. Fibroblast glutamate transport in aging and in $\mathrm{AD}$ : correlations with disease severity. Neurobiol. Aging. 26:825-832.

32. Tamura, H., et al. 2006. High frequency of openangle glaucoma in Japanese patients with Alzheimer's disease. J. Neurol. Sci. 246:79-83.

33. Libby, R.T., Gould, D.B., Anderson, M.G., and John, S.W. 2005. Complex genetics of glaucoma susceptibility. Annu. Rev. Genomics Hum. Genet. 6:15-44.

34. Dringen, R., Pfeiffer, B., and Hamprecht, B. 1999. Synthesis of the antioxidant glutathione in neurons: supply by astrocytes of CysGly as precursor for neuronal glutathione. J. Neurosci. 19:562-569.

35. Wu, G., Fang, Y.Z., Yang, S., Lupton, J.R., and Turner, N.D. 2004. Glutathione metabolism and its implications for health. J. Nutr. 134:489-492.

36. Reichelt, W., Stabel-Burow, J., Pannicke, T.,
Weichert, H., and Heinemann, U. 1997. The glutathione level of retinal Müller glial cells is dependent on the high-affinity sodium-dependent uptake of glutamate. Neuroscience. 77:1213-1224.

37. Riepe, R.E., and Norenburg, M.D. 1977. Müller cell localisation of glutamine synthetase in rat retina. Nature. 268:654-655.

38. Li, Q., and Puro, D.G. 2002. Diabetes-induced dysfunction of the glutamate transporter in retinal Müller cells. Invest. Ophthalmol. Vis. Sci. 43:3109-3116.

39. Ohia, S.E., Opere, C.A., and LeDay, A.M. 2005. Pharmacological consequences of oxidative stress in ocular tissues. Mutation Res. 579:22-36.

40. Lipton, S.A. 2001. Retinal ganglion cells, glaucoma and neuroprotection. Prog. Brain Res. 131:712-718.

41. Yamashita, H., Kawakami, H., Zhang, Y.X., Tanaka, K., and Nakamura, S. 1995. Neuroprotective mechanism of bromocriptine. Lancet. 346:1305.

42. Tanaka, K. 2005. Antibiotics rescue neurons from glutamate attack. Trends Mol. Med. 11:259-262.

43. Harada, T., et al. 2000. Modification of glialneuronal cell interactions prevents photoreceptor apoptosis during light-induced retinal degeneration. Neuron. 26:533-541.

44. Harada, T., et al. 2002. Microglia-Müller glia cell interactions control neurotrophic factor production during light-induced retinal degeneration. J. Neurosci. 22:9228-9236.

45. Ooto, S., et al. 2004. Potential for neural regeneration after neurotoxic injury in the adult mammalian retina. Proc. Natl. Acad. Sci. U. S. A. 101:13654-13659.

46. Harada, T., Harada, C., and Parada, L.F. 2007. Molecular regulation of visual system development: more than meets the eye. Genes Dev. 21:367-378.

47. Bringmann, A., et al. 2006. Müller cells in the healthy and diseased retina. Prog. Retin. Eye Res. 25:397-424.

48. Harada, C., Mitamura, Y., and Harada, T. 2006. The role of cytokines and trophic factors in epiretinal membranes: involvement of signal transduction in glial cells. Prog. Retin. Eye Res. 25:149-164.

49. Rothstein, J.D., et al. 1994. Localization of neuronal and glial glutamate transporters. Neuron. 13:713-725.

50. Fontaine, V., et al. 2002. Neurodegenerative and neuroprotective effects of tumor necrosis factor (TNF) in retinal ischemia: opposite roles of TNF receptor 1 and TNF receptor 2. J. Neurosci. 22:RC216.

51. Aihara, M., Lindsey, J.D., and Weinreb, R.N. 2002. Reduction of intraocular pressure in mouse eyes treated with latanoprost. Invest. Ophthalmol. Vis. Sci. 43:146-150.

52. Mitani, A., and Tanaka, K. 2003. Functional changes of glial glutamate transporter GLT-1 during ischemia: an in vivo study in the hippocampal CA1 of normal mice and of mutant mice lacking GLT-1. J. Neurosci. 23:7176-7182. 